\title{
Overview of Multiple-Aperture Interferometry Binary Star Results from the Southern Hemisphere
}

\author{
John Davis \\ School of Physics, University of Sydney, \\ NSW 2006, Australia \\ email: j.davis@physics.usyd.edu.au
}

\begin{abstract}
The first multiple-aperture interferometric study of a binary system, in which the power of combining interferometric and spectroscopic data was demonstrated, was made from the Southern Hemisphere. The observations of $\alpha$ Vir with the Narrabri Stellar Intensity Interferometer (NSII) were combined with spectroscopic and photometric data to yield the mass, radius and luminosity of the primary as well as an accurate distance to the system. The NSII also revealed a number of stars, previously thought to be single, to be binary systems. Several of these systems have subsequently been shown to be spectroscopic binaries.

The Sydney University Stellar Interferometer (SUSI) and the European Southern Observatory's Very Large Telescope Interferometer (VLTI) are the two current Southern Hemisphere multiple aperture interferometers. SUSI is being used to determine interferometric orbits for some of the binary systems discovered with the NSII including $\beta$ Cen and $\lambda$ Sco and, in combination with spectroscopy, to determine accurate masses for early-type stars and accurate dynamical parallaxes for the systems.

The VLTI has operated with three beam-combining instruments, namely VINCI, MIDI and AMBER. The few observations of binary systems that have been made so far are summarised and, while in general they are of a preliminary nature, they demonstrate the potential of the VLTI for binary star studies.

One double-lined spectroscopic binary that has been observed with all three Southern Hemisphere instruments is $\gamma^{2}$ Vel, which has the brightest Wolf-Rayet star in the sky as its secondary. The observations and preliminary results for the masses of the O-type primary and WC8 secondary and for the distance to the system are summarised.
\end{abstract}

Keywords. techniques: interferometric, techniques: spectroscopic, stars: binaries, stars: fundamental parameters, stars: distances

\section{Introduction}

The Narrabri Stellar Intensity Interferometer (NSII) (Hanbury Brown, Davis \& Allen 1967) was the first of the three Southern Hemisphere multiple-aperture optical interferometers. It represents an important milestone in the study of binary stars as it was the first long-baseline optical interferometer with independently controlled apertures to be used to determine the orbital parameters of a binary system and to demonstrate the power of combining interferometric and spectroscopic data.

The other two Southern Hemisphere multiple-aperture interferometers are the Sydney University Stellar Interferometer (SUSI) (Davis et al. 1999) and the European Southern Observatory's Very Large Telescope Interferometer (VLTI) (Glindemann et al. 2003). Each of the three instruments and the binary star results obtained with them will be discussed in turn as they generally differ in nature or in the types of system studied. 
Table 1. Parameters determined for $\alpha$ Vir from the combination of interferometric, spectroscopic and photometric data (Herbison-Evans et al. 1971). The symbols are defined in the text.

\begin{tabular}{|c|c|c|}
\hline Interferometry & \multicolumn{2}{|c|}{ Plus Spectroscopy } \\
\hline$i=65.9 \pm 1.8 \mathrm{deg}$ & $\bar{a}$ & $=(1.93 \pm 0.06) \times 10^{7} \mathrm{~km}$ \\
\hline$=1.54 \pm 0.05 \mathrm{mas}$ & $d$ & $=84 \pm 4 \mathrm{pc}$ \\
\hline$\theta_{\mathrm{LD} 1}=0.90 \pm 0.04 \mathrm{mas}$ & $R_{1}$ & $=8.1 \pm 0.5 R_{\odot}$ \\
\hline$\beta=6.4 \pm 1.0$ & & $\begin{array}{l}=10.9 \pm 0.9 M_{\odot} \\
=6.8 \pm 0.7 M_{\odot}\end{array}$ \\
\hline Plus Spectrophotometry & $\log L_{1} / L_{\odot}$ & $=4.17 \pm 0.10$ \\
\hline$\overline{T_{\mathrm{e} 1}}=22400 \pm 1000 \mathrm{~K}$ & $\log g_{1}$ & $=3.7 \pm 0.1$ \\
\hline
\end{tabular}

An exception is the case of $\gamma^{2}$ Vel since this system has been observed with all three instruments and the results will be discussed together in Section 5 .

\section{The Narrabri Stellar Intensity interferometer}

The NSII (1965-1972) was used to measure the angular diameters of 32 early-type stars (Hanbury Brown, Davis \& Allen 1974), to study the double-lined spectroscopic binaries $\alpha$ Vir (Section 2.1) and $\gamma^{2}$ Vel (Section 5), and to discover previously unsuspected binary systems (Section 2.2).

\section{1. $\alpha$ Virginis}

The double-lined spectroscopic binary $\alpha$ Vir (B1 III-IV+B3 V) was observed in 1966 and 1970 with the NSII at $\lambda 442 \mathrm{~nm}$ to determine an interferometric orbit for the 4.015 day period system (Herbison-Evans et al. 1971). The results were combined with spectroscopically determined parameters for the system based on observations by Struve \& Ebbighausen (1934) and Struve et al. (1958) to demonstrate, for the first time, the power of combining interferometric and spectroscopic observations of double-lined spectroscopic binaries for the determination of fundamental properties of stars.

The two techniques allow the determination of some orbital parameters in common, namely the period $P$, the longitude of periastron $\omega$, and the eccentricity $e$. However, there are also complementary parameters: interferometry provides the inclination of the orbit $i$, the angular size of the semi-major axis $\theta_{\mathrm{a}}$, the brightness ratio of the two components $\beta$, and the angular size of at least the primary component of the system $\theta_{\mathrm{LD} 1}$ (limb darkened), whereas spectroscopy provides $a \sin i, M_{1} \sin ^{3} i$, and $M_{2} \sin ^{3} i$ where $a$ is the semi-major axis of the system and $M_{1}$ and $M_{2}$ are the masses of the component stars.

Full details have been given by Herbison-Evans et al. (1971) and some of the results are given in Table 1 to illustrate the importance of the combination of the two techniques. The addition of measurements of the spectral flux distribution allows the effective temperature $T_{\mathrm{e} 1}$ and luminosity $L_{1}$ of the primary to be determined.

The Hipparcos distance for $\alpha$ Vir is $80.4 \pm 5.5 \mathrm{pc}$ in good agreement with the NSII value of $84 \pm 4 \mathrm{pc}$.

\subsection{Discovered Binaries}

A by-product of the NSII programme was the discovery that several stars, previously thought to be single, were in fact binary systems (Hanbury Brown, Davis \& Allen 1974) but the instrument lacked the capability for determining their orbits. These included $\beta$ Cen, $\lambda$ Sco, $\delta$ Vel, $\zeta$ Ori, $\beta$ Cru, $\sigma$ Sgr and $\delta$ Sco. Each of these systems has subsequently had its binary nature confirmed by spectroscopy and/or interferometry and the first three will be discussed in more detail below. 


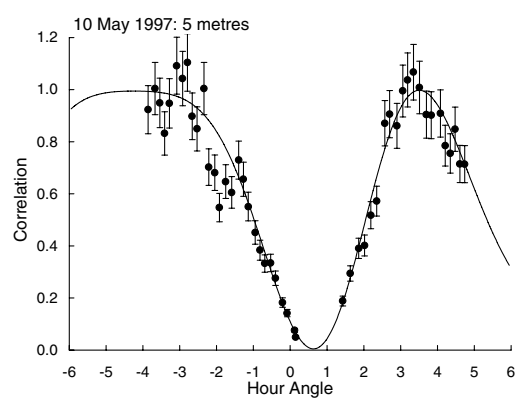

(a)

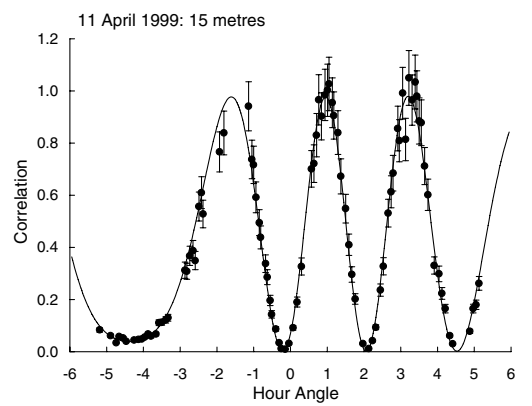

(c)

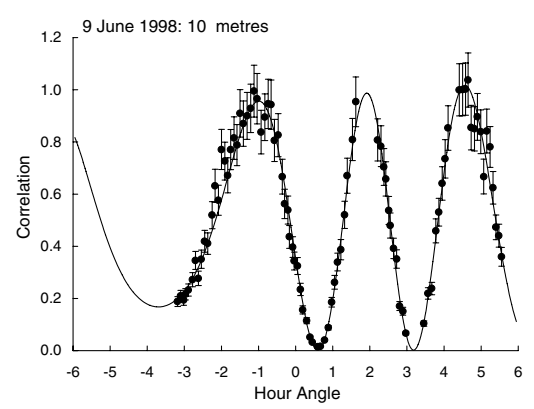

(b)

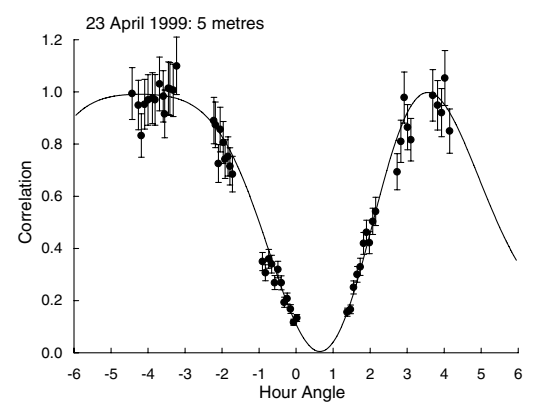

(d)

Figure 1. Normalised correlation versus hour angle for $\beta$ Cen on (a) May 10, 1997 at a baseline of $5 \mathrm{~m}$, (b) June 9, 1998 at $10 \mathrm{~m}$, (c) April 11, 1999 at $15 \mathrm{~m}$, and (d) April 23, 1999 at $5 \mathrm{~m}$. In each case the filled circles represent the measurements and the line is the fit to determine the vector separation of the components.

\section{The Sydney University Stellar Interferometer}

SUSI is a long baseline optical interferometer with a $640 \mathrm{~m}$ long North-South array of input siderostats, the light from any two of which can be combined at a time. Baselines from 5-160 m are fully operational and the spectral range of the instrument is $430-950 \mathrm{~nm}$. It has been used to observe a number of binary systems including $\beta$ Cen and $\lambda$ Sco discussed below, both discovered to be binaries with the NSII, and $\gamma^{2}$ Vel to be discussed in Section 5.

\section{1. $\beta$ Centauri}

The bright southern binary $\beta$ Cen was observed with SUSI at $\lambda 442 \mathrm{~nm}$ from $1997-2002$ to determine an interferometric orbit (Davis et al. 2005). The component stars are of almost equal brightness $(\Delta \mathrm{m}$ at $\lambda 442 \mathrm{~nm}$ is $0.15 \pm 0.02)$ and of spectral type B1 III. Observations were made over a range in hour angle for each observing night to enable vector separations to be determined. Four examples are shown in Figure 1 and inspection shows that the plots for 10 May 1997 and 23 April 1999, both obtained with a baseline of $5 \mathrm{~m}$, are almost identical. This is to be expected since they are separated in time by $1.9973 \pm 0.0004$ orbital periods. The orbit fitted to the vector separations is shown in Figure 2.

The interferometric results were combined by Davis et al. (2005) with a revision of the spectroscopic results of Ausseloos et al. (2002) and the results are summarised in Table 2. There are two points to note about the parameters listed in Table 2. Firstly, Ausseloos et al. (2006) have improved the disentangling of the spectral lines of the component stars of $\beta$ Cen, resulting in a revision of the parameters in the table. Using the same approach as Davis et al. (2005) with the revised spectroscopic data led to masses of 


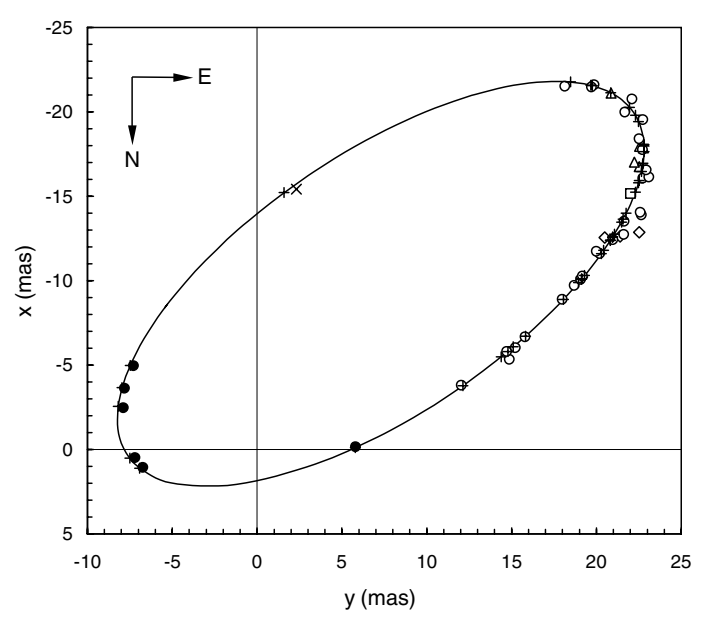

Figure 2. The orbit of $\beta$ Centauri. The data points are SUSI results except for the MAPPIT point which is from Robertson et al. (1998). SUSI observations: $1997 \diamond ; 1998 \triangle ; 1999$ O; 2000 -; $2002 \square$. MAPPIT observation: 1995 X. The points on the fitted orbit that correspond to the observational points are marked with + .

$M_{1}=11.2 \pm 0.7 M_{\odot}$ and $M_{2}=9.8 \pm 0.7 M_{\odot}$ with a reversal of the identification of primary and secondary. The dynamical parallax was revised to $\pi_{\mathrm{d}}=9.3 \pm 0.3$ mas. The uncertainties have been doubled in a conservative approach because the uncertainty in the disentangling process could not be propagated directly. The mass estimates were further refined using additional observational information and the extensive database of main-sequence stellar models by Ausseloos et al. (2004) to give $M_{1}=10.7 \pm 0.1 M_{\odot}$ and $M_{2}=10.3 \pm 0.1 M_{\odot}$. The second point to note is that the dynamical parallax differs significantly from the Hipparcos value of $\pi=6.21 \pm 0.56$ mas. It is thought to be due to the fact that the binary nature of $\beta$ Cen, with its relatively large variation in the projected angular separation of the two components on the sky, was not taken into account in determining the Hipparcos parallax.

Table 2. Parameters determined for $\beta$ Cen taken from Davis et al. (2005). The three left-hand columns list values determined from spectroscopy, identified by $\mathrm{S}$ in the third column, and from interferometry, identified by I. The right-hand columns list parameters determined by the combination of the interferometric and spectroscopic data. $P, a, a^{\prime \prime}$ and $i$ represent the period, semi-major axis, angular semi-major axis and inclination of the orbit respectively. $\beta$ is the brightness ratio of the two components determined from the interferometric observations. $\pi_{d}$ is the dynamical parallax.

\begin{tabular}{lrcc|lll}
\hline \multicolumn{1}{c}{ Parameter } & \multicolumn{2}{c}{ Value } & \multicolumn{2}{c}{ Source } & Parameter & \multicolumn{2}{c}{ Value } \\
\hline$P($ days $)$ & $357.00 \pm 0.07$ & $\mathrm{~S}$ & $\pi_{\mathrm{d}}(\mathrm{mas})$ & $9.77 \pm 0.15$ \\
$M_{1} \sin ^{3} i\left(M_{\odot}\right)$ & $7.15 \pm 0.24$ & $\mathrm{~S}$ & $M_{1}\left(M_{\odot}\right)$ & $9.09 \pm 0.31$ \\
$M_{2} \sin ^{3} i\left(M_{\odot}\right)$ & $7.15 \pm 0.23$ & $\mathrm{~S}$ & $M_{2}\left(M_{\odot}\right)$ & $9.09 \pm 0.30$ \\
$a \sin i(\mathrm{AU})$ & $2.391 \pm 0.032$ & $\mathrm{~S}$ & $\mathrm{M}_{1 V}$ & $-3.85 \pm$ & \pm 0.05 \\
$a^{\prime \prime}$ & $0.02532 \pm 0.00023$ & $\mathrm{I}$ & $\mathrm{M}_{2 \mathrm{~V}}$ & $-3.70 \pm 0.05$ \\
$i(\mathrm{deg})$ & $67.4 \pm 0.3$ & $\mathrm{I}$ & & & & \\
$\beta($ at $442 \mathrm{~nm})$ & $0.868 \pm 0.015$ & $\mathrm{I}$ & & & \\
\hline
\end{tabular}


Table 3. Parameters determined for $\lambda$ Sco with SUSI (from Tango et al. 2006). The two left-hand columns list values determined interferometrically with SUSI and the right-hand columns list parameters determined by the combination of the interferometric and spectroscopic data. $P, a^{\prime \prime}$ and $i$ represent the period, angular semi-major axis and inclination of the orbit respectively. The $\Delta \mathrm{m}$ values are the magnitude differences at the specified wavelengths determined from the interferometric observations. $M_{1}$ and $M_{2}$ are the masses of the components and $\pi_{d}$ is the dynamical parallax.

\begin{tabular}{|c|c|c|c|c|}
\hline Parameter & Value & | Parameter & Value & Spectral Type \\
\hline$P$ (days) & $1052.8 \pm 1.2$ & $M_{1}\left(M_{\odot}\right)$ & $10.4 \pm 1.3$ & B1.5 IV \\
\hline$a^{\prime \prime}$ & $0.0493 \pm 0.0003$ & $M_{2}\left(M_{\odot}\right)$ & $8.1 \pm 1.0$ & B2 IV \\
\hline$i(\operatorname{deg})$ & $77.2 \pm 0.2$ & $\pi_{\mathrm{d}}$ (mas) & $8.9 \pm 0.4$ & \\
\hline$\Delta \mathrm{m}_{442}$ & $0.65 \pm 0.10$ & & & \\
\hline$\Delta \mathrm{m}_{700}$ & $0.67 \pm 0.10$ & & & \\
\hline
\end{tabular}

\section{2. $\lambda$ Scorpii}

$\lambda$ Sco is a well-known single-lined spectroscopic binary with a $\beta$ Cep primary and a low mass companion orbiting it with a period of $\sim 6$ days. The detection of a third component by the NSII has been confirmed by radial velocity measurements (Uytterhoeven et al. 2004) and by SUSI. The third component is a B star orbiting the primary with a period of $\sim 2.9$ years and the interferometric orbit determined with SUSI (Tango et al. 2006) is shown in Figure 3. Tango et al. (2006) have combined the SUSI results with the spectroscopic results of Uytterhoeven et al. (2004), revised using the more accurate values for $P, T, e$ and $\omega$ from the interferometry, and these are summarised in Table 3.

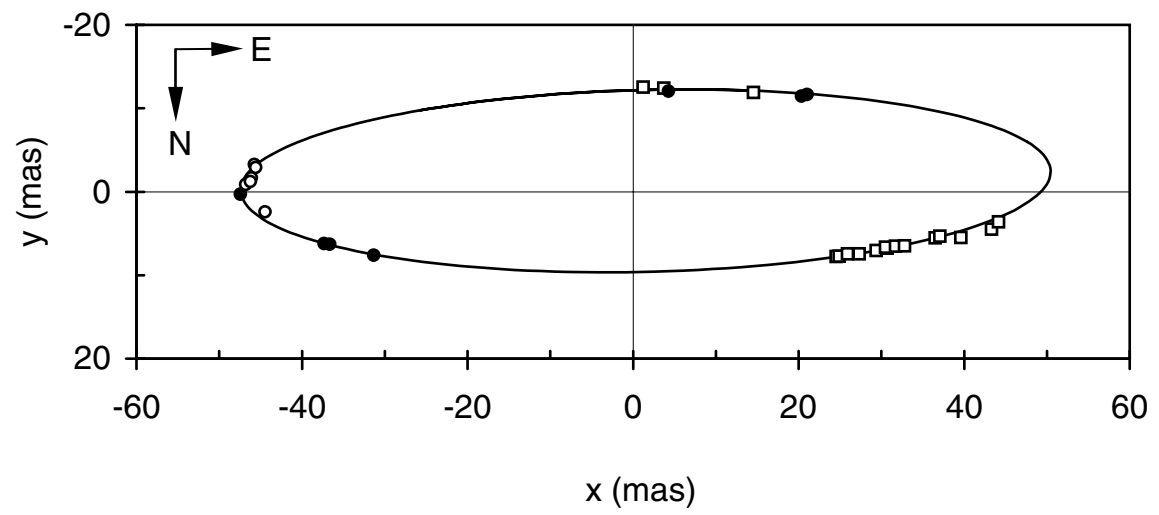

Figure 3. The orbit of $\lambda$ Sco determined with SUSI. Key: $\bigcirc 1999$ observations; $\square 2000$ and 2001 observations; 2004 and 2005 observations made during the next orbit. The 1999-2001 observations were made at a wavelength of $442 \mathrm{~nm}$ and the 2004-5 observations at $700 \mathrm{~nm}$.

As in the case of $\beta$ Cen, the dynamical parallax for $\lambda$ Sco differs significantly from the Hipparcos value of $\pi=4.64 \pm 0.90$ mas and it is thought to be for the same reason.

\subsection{SUSI Summary}

A major component of the SUSI observational programme is the observation of spectroscopic binary stars to determine stellar masses and dynamical parallaxes as demonstrated for $\beta$ Cen and $\lambda$ Sco. $\gamma^{2}$ Vel is discussed in Section 5. Several systems are in the programme including the 33 day period double-lined spectroscopic binary $\sigma$ Sco, another system with a $\beta$ Cep component. 


\section{ESO's Very Large Telescope Interferometer}

ESO's VLTI (Glindemann et al. 2003) was commissioned with two test siderostats of $0.4 \mathrm{~m}$ diameter and baselines in the range 8-202 $\mathrm{m}$. It also has the capability of using any of the four $8.2 \mathrm{~m}$ Unit Telescopes (UTs) with baselines in the range $47-130 \mathrm{~m}$ and, more recently, four $1.8 \mathrm{~m}$ Auxiliary Telescopes (ATs) with the same baseline range as the test siderostats. Three beam-combining instruments have been used with the VLTI to date, namely VINCI, MIDI and AMBER.

\subsection{VINCI}

VINCI (Kervella et al. 2003) was the commissioning instrument for the VLTI and worked in the $\mathrm{K}$ band $(2.0-2.4 \mu \mathrm{m})$. It combined the light from either the two test siderostats or from two UTs. It was primarily a test instrument and many observations were made of single stars including Cepheids and a rapidly rotating star. However, the only binary star observations were of $\delta$ Vel.

The primary of $\delta$ Vel was found to be a binary system with the NSII (Hanbury Brown et al. 1974) and it has subsequently been found to be a 45 day period eclipsing system (Otero, Fieseler \& Lloyd 2000). $\delta$ Vel is therefore a triple system in which the primary $(\mathrm{Aa}+\mathrm{Ab})$ is the eclipsing system. Aa and Ab are classified as A2 and A4 and the B component as F2/5. Observations of the eclipsing system were made at four orbital phases in 2003 with VINCI and at one orbital phase in 2005 with AMBER. It has been found that not all the VINCI data fit a simple model and there are significant discrepancies (A. Kellerer, private communication). The conclusion to be drawn is that more data are needed before a satisfactory solution for this system can be established.

\section{2. $M I D I$}

MIDI (Leinert et al. 2003) is a two aperture beam-combining instrument working in the $\mathrm{N}$ band $(7.8-13.5 \mu \mathrm{m})$. Observations have been made of a number of binary systems containing T-Tauri stars (Ratzka \& Leinert 2007). The emphasis has been on the study of disks associated with widely spaced component stars such as VV CrA (Ratzka et al. 2007). Essentially this has been a study of the disks rather than of the binaries themselves.

Vector separations have been measured for Z CMa (Ratzka \& Leinert 2007) in good agreement with adaptive optics measurements, and for FU Ori (Quanz et al. 2006) in good agreement with earlier work . These measurements, although not providing new results, are a confirmation of the performance of the VLTI-MIDI combination and demonstrate its capability.

MIDI has also been used to observe the disks around the cores of planetary nebulae. Arguments have been advanced to suggest that the formation of the disks and their chemistry are caused by the presence of binary companions. Although binary companions have not been detected from the limited observations to date, the MIDI data and their interpretation have been discussed by Matsuura et al. (2006) and Chesneau et al. (2006).

Observations of some stars with MIDI have revealed circumstellar disks that are smaller than expected and this has led to the suspicion that the disks are being truncated by the presence of unseen companions - an example is the Be star $\alpha$ Ara (Chesneau et al. 2005).

To summarize the work so far with the relatively new MIDI instrument, most of the observational data have not been fully interpreted and in some cases more data are needed. A fair summary is to say that observations made with MIDI are mainly work in progress but that its potential for the study of young stars and disks in binary systems has been clearly demonstrated. 
Table 4. Orbital parameters for $\gamma^{2}$ Vel: NSII values from Hanbury Brown et al. (1970), spectroscopic values from Schmutz et al. (1997), and SUSI values are preliminary results from work in progress. Values marked with an asterisk are the values adopted and fixed in the orbital solution for the interferometric data.

\begin{tabular}{lccc}
\hline Parameter & NSII & Spectroscopy & SUSI \\
\hline$P$ (days) & $78.5^{*}$ & $78.53 \pm 0.01$ & $78.53^{*}$ \\
$\theta_{\mathrm{a}}$ (mas) & $4.3 \pm 0.5$ & & 3.6 \\
$e$ & $0.17 \pm 0.03$ & $0.326 \pm 0.010$ & 0.33 \\
$\mathrm{~T}(\mathrm{MJD})$ & & $50120 \pm 2$ & 50121 \\
$\omega(\mathrm{deg})$ & $267 \pm 9^{*}$ & $248 \pm 4$ & 247.3 \\
$\Omega(\mathrm{deg})$ & & & \\
$i(\mathrm{deg})$ & $70^{*}$ & $65 \pm 8$ & 65.8 \\
\hline
\end{tabular}

\section{3. $A M B E R$}

AMBER (Petrov et al. 2003) is a three aperture beam-combining instrument covering the $\mathrm{J}, \mathrm{H}$ and $\mathrm{K}$ bands with the capability of recording spectra, dispersed fringes and closure phases. It is early in the life of the instrument and, apart from some early observations of $\delta$ Vel mentioned in Section 4.1, the only binary studied so far is $\gamma^{2}$ Vel and these observations will be discussed in Section 5 .

Observations of $\eta$ Car with AMBER, primarily aimed at studying the wavelength dependence of the optically thick wind region with high spatial and spectral resolution, have also been used to investigate the detectability of a hypothetical hot binary companion (T. Gull - private communication). While not directly detecting the companion, it is hoped that additional AMBER observations of higher accuracy will be sensitive enough to establish its existence.

Future plans for the VLTI-AMBER combination include observations of eclipsing binaries for distance determinations (A. Richichi - private communication).

\section{5. $\gamma^{2}$ Velorum}

$\gamma^{2}$ Vel is a double-lined spectroscopic binary system containing the brightest WolfRayet star in the sky and it is therefore of particular interest. The primary is an $\mathrm{O}$ star with spectral classifications that range from O7 III to O9 I and the secondary is the WC8 Wolf-Rayet star. The system has been observed with the NSII, SUSI and VLTI-AMBER.

The first interferometric observations were made with the NSII in 1968 (Hanbury Brown et al. 1970). Observations were made in the continuum at $443 \mathrm{~nm}$ and in the C III-IV emission lines at $465 \mathrm{~nm}$. At the time the Wolf-Rayet star was thought to be the primary and so the interpretation of the $443 \mathrm{~nm}$ results for the Wolf-Rayet star actually referred to the $\mathrm{O}$ star but the value determined for the angular semi-major axis of the orbit of $4.3 \pm 0.5$ mas is valid. The $465 \mathrm{~nm}$ observations were aimed at establishing the relative size of the CIII-IV emission region around the Wolf-Rayet star but need to be repeated with the new knowledge of the system and this is planned for SUSI.

Observations with SUSI at $700 \mathrm{~nm}$ and a baseline of $80 \mathrm{~m}$ have been made to determine the orbit for $\gamma^{2}$ Vel and preliminary results are listed in Table 4. Also listed in Table 4 are the NSII results and spectroscopic results from Schmutz et al. (1997).

$\gamma^{2}$ Vel was observed in the dispersed K band on 25 December 2004 with the VLTIAMBER combination using three UTs to give a range of interferometric and spectroscopic data (Petrov et al. 2007, Malbet et al. 2006, and R. Petrov, private communication). Although it yielded only a data set for a single night with limited u-v coverage it illustrated 
Table 5. Fundamental quantities for $\gamma^{2}$ Velorum: NSII values from Hanbury Brown et al. (1970), SUSI values and uncertainties are preliminary results from work in progress, the AMBER distance is from Malbet et al. (2007) and the Hipparcos distance (d) is from Schaerer et al. (1997). The values in parentheses have been derived from the published data.

\begin{tabular}{lcccc}
\hline \multicolumn{1}{c}{ Parameter } & NSII & SUSI & AMBER & Hipparcos \\
\hline$M(\mathrm{O}) / M_{\odot}$ & \multicolumn{5}{c}{$28.6 \pm 1.5$} \\
$M(\mathrm{WR}) / M_{\odot}$ & $9.0 \pm 0.8$ & & \\
$\pi(\mathrm{mas})$ & $(2.9 \pm 0.4)$ & $2.96 \pm 0.16$ & $(2.72 \pm 0.37)$ & $3.88 \pm 0.53$ \\
$\mathrm{~d}(\mathrm{pc})$ & $350 \pm 50$ & $338 \pm 20$ & $368_{-13}^{+38}$ & $258_{-31}^{+41}$ \\
$\mathrm{M}_{\mathrm{V}}(\mathrm{O})$ & -5.6 & -5.7 & $(-5.9)$ & $(-5.1)$ \\
$\mathrm{M}_{\mathrm{V}}(\mathrm{WR})$ & -4.6 & -4.4 & $(-4.6)$ & $(-3.8)$ \\
\hline
\end{tabular}

the potential of AMBER to provide spatial resolution for close binaries. In summary, one vector separation has been determined from the observations with the $180^{\circ}$ ambiguity present for a two-aperture interferometer removed. Modelling of the spectra based on a simple geometrical binary model has been carried out giving a reasonable but not fully consistent fit with the observed data. The distance to the system has been determined from the AMBER data and this is listed in Table 5.

The distance has also been determined by combining the preliminary SUSI results with the spectroscopic results of Schmutz et al. (1997) to establish preliminary values for the masses of the component stars and the distance to the system and these are listed in Table 5 with the absolute visual magnitudes of the components.

In Table 5 the values shown in parentheses have been derived from the published results. The preliminary SUSI distance is in good agreement with the NSII and AMBER results and the following conclusions can be reached. The system is intrinsically brighter by a factor of $\sim 1.7$ than given by the Hipparcos parallax. The accuracy of the SUSI mass determinations, although not final values, shows that the accuracy is limited by the spectroscopic data. The separation of the spectra of the component stars and the determination of their relative flux contributions is a matter for further work.

\section{Summary}

The main contributions to binary star studies with multiple-aperture interferometry from the Southern Hemisphere have been by the NSII ( $\alpha$ Vir) and currently by SUSI. SUSI is contributing mass determinations for early type stars including $\beta$ Cep types in collaboration with Belgian spectroscopists. ESO's VLTI has yet to make a major contribution to binary star research but it has great potential as demonstrated by the observations of young binary systems, particularly those involving disks, with the N-band MIDI instrument and by early observations with the AMBER instrument in the K-band which, with its dispersed fringes, closure phase and imaging possibilities, has demonstrated its potential for studies of close binary systems. The planned programmes for SUSI and the VLTI are significantly different, particularly in the wavelength ranges covered but also, to a large extent, in the types of system observed. For these reasons the two instruments can be regarded as complementary for Southern Hemisphere interferometric binary star studies.

\section{Acknowledgements}

I would like to acknowledge my SUSI colleagues for the assistance they have given me in preparing this review and, in particular, Julian North for providing me with the 
preliminary SUSI results for $\gamma^{2}$ Vel. I also acknowledge information on the status of binary star observations with the VLTI that I have received from Olivier Chesneau, Aglaé Kellerer, Christoph Leinert, Romain Petrov, Thorsten Ratzka, Andrea Richichi and Markus Schöller.

\section{References}

Ausseloos, M., Aerts, C., Uytterhoeven, K., Schrijvers, C., Waelkens, C., \& Cuypers, J. 2002, $A \mathscr{E} A 384,209$

Ausseloos, M., Aerts, C., Lefever, K., Davis, J., \& Harmanec, P. 2006, A\&A 4 455, 259

Ausseloos, M., Scuflaire, R., Thoul, A., \& Aerts, C. 2004, MNRAS 355, 352

Chesneau, O. et al. 2005, A\&SA 435, 275

Chesneau, O. et al. 2006, A\&BA 455, 1009

Davis, J., Tango, W.J., Booth, A.J., ten Brummelaar, T.A., Minard, R.A., \& Owens, S.M. 1999, MNRAS 303, 773

Davis, J., Mendez, A., Seneta, E.B., Tango, W.J., Booth, A.J., O'Byrne, J.W., Thorvaldson, E.D., Ausseloos, M., Aerts, C., \& Uytterhoeven, K. 2005, MNRAS 356, 1362

Glindemann, A. et al. 2003, in: W.A. Traub (ed.) Interferometry for Optical Astronomy II, Proceedings of SPIE Vol. 4838, p. 89

Hanbury Brown, R., Davis, J., \& Allen, L.R. MNRAS 1967, 137, 375

Hanbury Brown, R., Davis, J., \& Allen, L.R. MNRAS 1974, 167, 121

Hanbury Brown, R., Davis, J., Herbison-Evans, D., \& Allen, L.R. MNRAS 1970, 148, 103

Herbison-Evans, D., Hanbury Brown, R., Davis, J., \& Allen, L.R. MNRAS 1971, 151, 161

Kervella, P. et al. 2003, in: W.A. Traub (ed.) Interferometry for Optical Astronomy II, Proceedings of SPIE Vol. 4838, p. 858

Leinert, C. et al. 2003, in: W.A. Traub (ed.) Interferometry for Optical Astronomy II, Proceedings of SPIE Vol. 4838, p. 893

Malbet, F., Petrov, R.G., Weigelt, G., Stee, P., Tatulli, E., Domiciano de Souza, A., \& Millour, F. and the AMBER consortium 2006, in J.D. Monnier, M. Schöller, W.C. Danchi (eds.) Advances in Stellar Interferomety, Proceedings of SPIE Vol. 6268, CID 626802

Matsuura, M. et al. 2006, ApJ 646, 123

Otero, S.A., Fieseler, P.D., \& Lloyd, C. 2000, IAU Information Bulletin on Variable Stars, No. 4999 (Konkoly Observatory, Budapest)

Petrov, R.G. et al. 2003, in: W.A. Traub (ed.) Interferometry for Optical Astronomy II, Proceedings of SPIE Vol. 4838, p. 924

Petrov, R. et al. 2007, in: F. Paresce \& A. Richichi (eds.), The Power of Optical/IR Interferometry, Proc. ESO Workshop (Berlin: Springer-Verlag), in press

Quanz, S.P., Henning, Th., Bouwman, J., Ratzka, Th., \& Leinert, Ch. 2006, ApJ 648, 472

Ratzka, Th., Leinert, Ch. 2007, in: F. Paresce \& A. Richichi (eds.), The Power of Optical/IR Interferometry, Proc. ESO Workshop (Berlin: Springer-Verlag), in press

Ratzka, Th., Leinert, Ch., Przygodda, F., \& Wolf, S. 2007, in: F. Paresce \& A. Richichi (eds.), The Power of Optical/IR Interferometry, Proc. ESO Workshop (Berlin: Springer-Verlag), in press

Robertson, J.G., Bedding, T.R., Aerts, C., Waelkens, C., Marson, R.G., \& Barton, J.R. MNRAS 1999, 302, 245

Schaerer, D., Schmutz, W., \& Grenon, M. 1997, ApJ 484, L153

Schmutz, W., Schweickhardt, J., Stahl, O., Wolf, B., Dumm, T., Gäng, Th., Jankovics, I., Kaufer, A., Lehmann, H., Mandel, H., Peitz, J., \& Rivinius, Th. 1997, A\& A 328, 219

Struve, O., Ebbighausen, E. 1934, ApJ 80, 365

Struve, O., Sahade, J., Huang, S-S., Zebergs, V. 1958, ApJ 128, 310

Tango, W.J., Davis, J., Ireland, M.J., Aerts, C., Uytterhoeven, K., Jacob. A.P., Mendez, A., North, J.R., Seneta, E.B., \& Tuthill, P.G. 2006, MNRAS 370, 884

Uytterhoeven, K., Willems, B., Lefever, K., Aerts, C., Telting, J.H., \& Kolb, U. 2004, A\& $A 427$, 581 